\title{
Novel TMEM127Variant Associated to Bilateral Phaeochromocytoma with an Uncommon Clinical Presentation
}

\author{
Antía Fernández-Pombo (iD, ${ }^{1,2}$ José M. Cameselle-Teijeiro ${ }^{(D)}{ }^{3}$ Jose A. Puñal-Rodríguez, ${ }^{4}$ \\ Lourdes Loidi, ${ }^{5}$ Roberto Peinó-García, ${ }^{1}$ Paloma Cabanas-Rodríguez, ${ }^{6}$ \\ Miguel Garrido-Pumar, ${ }^{7}$ Sandra Baleato-González, ${ }^{8}$ Enrique Flores-Ríos, ${ }^{8}$ \\ and David Araújo-Vilar (iD)
}

${ }^{1}$ Division of Endocrinology, University Clinical Hospital of Santiago de Compostela, Spain

${ }^{2}$ UETeM-Molecular Pathology Group, Department of Medicine, IDIS-CIMUS, University of Santiago de Compostela, Spain

${ }^{3}$ Division of Pathology, University Clinical Hospital of Santiago de Compostela, Spain

${ }^{4}$ Division of Surgery, University Clinical Hospital of Santiago de Compostela, Spain

${ }^{5}$ Fundación Galega de Medicina Xenómica, Santiago de Compostela, Spain

${ }^{6}$ Division of Pediatrics, University Clinical Hospital of Santiago de Compostela, Spain

${ }^{7}$ Division of Nuclear Medicine, University Clinical Hospital of Santiago de Compostela, Spain

${ }^{8}$ Division of Radiology, University Clinical Hospital of Santiago de Compostela, Spain

Correspondence should be addressed to David Araújo-Vilar; david.araujo@usc.es

Received 21 May 2019; Accepted 3 September 2019; Published 27 October 2019

Academic Editor: Carlo Capella

Copyright ( 2019 Antía Fernández-Pombo et al. This is an open access article distributed under the Creative Commons Attribution License, which permits unrestricted use, distribution, and reproduction in any medium, provided the original work is properly cited.

Phaeochromocytomas and paragangliomas are rare catecholamine-secreting tumours arising from the adrenal medulla or sympathetic paraganglia. It is known that $20-30 \%$ of all cases occur as a result of germline variants in several well known genes. The TMEM127 gene was recently identified as a new phaeochromocytoma susceptibility gene. However, until a larger sample of cases is available, the prevalence, genotype-phenotype correlation, and a clear predominant biochemical pattern of TMEM127-related PCC, remain to be defined. We present a woman with the pathogenic variant c.86delG (p.Arg29Leufs ${ }^{*}$ 52) in the TMEM127 gene, which has not been previously reported, associated to a bilateral phaeochromocytoma, with an uncommon initial clinical presentation and a biochemical profile that is distinctly adrenergic. Her two young children carry the same variant and are, at present, disease-free. Physicians should be aware that phaeochromocytoma can manifest in an atypical manner, as with episodic hypotension, mainly if the symptoms have no obvious aetiology and they worsen over time. This case also supports the presence of a predominant adrenaline secreting pattern in TMEM127-positive tumours, as well as the need to consider multigene panel testing in patients with bilateral phaeochromocytomas.

\section{Introduction}

Phaeochromocytomas (PCC) and paragangliomas are rare neural crest-derived tumours found respectively in the adrenal medulla or sympathetic paraganglia [1]. These tumours are usually benign and sporadic. However, $20-30 \%$ of all cases are part of well characterized hereditary tumour syndromes [2], such as von Hippel-Lindau disease, Multiple Endocrine Neoplasia type 2 and Neurofibromatosis type 1, which occur as a result of germline variants in VHL, RET,
NF1, and $S D H$ (subunits A, B, C, D and AF2) genes [3]. In addition, in 2010-2011, the TMEM127 and MAX genes were also identified as new PCC susceptibility genes $[4,5]$. The TMEM127 gene encodes a transmembrane protein that is linked to the regulation of the mTOR signaling complex. The majority of the variants described so far are associated with truncation of the protein, suggesting that they result in loss of TMEM127 function [4]. Here, we present a family with a novel pathogenic variant in the TMEM127 gene, associated to a bilateral PCC. 


\section{Case Presentation}

A 33-year-old woman first consulted at the Division of Cardiology because of presyncopal symptoms, which started just after the delivery of her first child, with acral pallor, associated with pain, tremor and weakness in the lower limbs. In addition, she presented gastrointestinal symptoms with epigastric pain and nausea, accompanied by slow palpitations and significant orthostatic hypotension. During one of the episodes an electrocardiogram showed a nodal rhythm with a ventricular rate of 50 . The Holter revealed a clear relation between the symptomatology and these episodes of nodal rhythm. Seven years later, after a routine blood test she showed an impaired fasting glucose of $110 \mathrm{mg} / \mathrm{dl}$ and was referred to the Division of Endocrinology. During a thorough anamnesis, the patient reported worsening of the symptoms and an increase in their frequency, with the appearance of pulsating headaches, dizziness, tinnitus and high blood pressure (up to $225 / 117 \mathrm{mmHg}$ ) followed by hypotension $(63 / 36 \mathrm{mmHg}$ on average). As far as her medical history is concerned, she had undergone a thyroid lobectomy with isthmusectomy, with a histological finding of a nodular hyperplasia. There was no family history of endocrine disease. However, her mother had hypertension and her father passed away due to a brainstem stroke. She has two healthy children.

With the clinical suspicion of PCC, an analytical study was requested, which showed: urine adrenalin $367 \mathrm{mcg} / 24 \mathrm{~h}$ $(<20 \mathrm{mcg} / 24 \mathrm{~h})$, urine noradrenalin $81 \mathrm{mcg} / 24 \mathrm{~h}$ (15$80 \mathrm{mcg} / 24 \mathrm{~h}$ ), urine metanephrine $2490 \mathrm{mcg} / 24 \mathrm{~h}$ (25$312 \mathrm{mcg} / 24 \mathrm{~h}$ ) and urine normetanephrine $816 \mathrm{mcg} / 24 \mathrm{~h}$ $(60-757 \mathrm{mcg} / 24 \mathrm{~h})$. Magnetic resonance imaging (MRI) (Figure 1) revealed bilateral adrenal masses, $37 \mathrm{~mm}$ right and $49 \mathrm{~mm}$ left. It showed a hyperintense signal of both adrenal glands on fat-suppressed T2-weighted, without the presence of fat. After gadolinium administration these masses enhanced avidly and heterogeneously with regions of no enhancement due to cystic changes. Restricted diffusion $(b=800)$ of hypercellular areas was well demonstrated by ADC map as areas of low intensity signal. In oppossite, cystic components demonstrated high signal. ${ }^{123}$ I-MIBG SPECT/CT (Figure 2) showed increased uptake foci in both adrenal glands, with right predominance, compatible with the diagnosis of bilateral PCC. No extraadrenal tumour localization was detected.

The patient underwent a laparoscopic bilateral adrenalectomy. The bilateral tumours were circumscribed but unencapsulated, and partially surrounded by a bright-yellow ring of normal adrenal cortical cells. Both tumours also showed a fleshy, tan cut surface, with haemorrhage areas, measuring $4.5 \mathrm{~cm}$ and $5.4 \mathrm{~cm}$ maximum diameter in the right and left glands respectively (Figure 3 ).

Genetic testing was carried out. The patient presented the pathogenic variant NM_017849.3: c.86delG (p.Arg29Leufs* $52)$ in heterozygosis in the TMEM127 gene. This variant has not been reported in available databases (i.e., dbSNP146, 1000 Genomes, ExAC and GnomAD). This consists of the deletion of one nucleotide in exon 2 of TMEM127 which alters the reading pattern and leads to the appearance of a premature stop codon. Given that this premature stop codon is 7 nucleotides upstream the next exon-junction complex, it is unlikely that this new transcript is down regulated by nonsense-mediated decay [6]. This result suggests a susceptibility to PCC with an autosomal dominant inheritance pattern (OMIM \#171300). Given these findings, a genetic study was performed on her children, aged 9 and 6, who were proved to be carriers of the same variant in the TMEM127 gene. Plasma and urine catecholamines and metanephrines and total body MRI showed normal results in both children. However, given the risk of developing PCC or other tumours (renal carcinoma) [7], they will require surveillance. In addition, her mother tested negative for the variant.

One year after the adrenalectomy the patient is asymptomatic, with normal metanephrine excretion. As expected, she has primary adrenal insufficiency and is being treated with hydrocortisone.

\section{Discussion}

To the best of our knowledge, this is the first report of a Spanish patient showing that a TMEM127 variant plays a pathological role in bilateral PCC. Furthermore, this c.86delG variant has not been previously reported.

Patients carrying TMEM127 pathogenic variants have a similar age of tumour diagnosis as those who have sporadic lesions (41.5 years vs. 45 years); one-third have bilateral disease; the tumours are usually benign; the occurrence of catecholamine excess with symptoms precede PCC diagnosis by several years, and only $25 \%$ have a family history of PCC [4, $8,9]$. In this case, in spite of its paroxysmal occurrence, indicative of PCC, the atypical clinical scenario caused a delay in the diagnosis. It is necessary to consider that the presence of sinus node dysfunction may be an initial manifestation of PCC [10]. Physicians must also be aware that it may likewise present with hypotension (excessive stimulation of beta adrenoceptors by elevated levels of epinephrine), postural hypotension or alternating episodes of high and low blood pressure [11]. However, a clear genotype-phenotype correlation is unlikely to become evident until a larger sample of these families is published. It has also been suggested that these patients can likewise develop cathecholamine-mediated arterial vasospasm, leading to cerebral ischemia [12]. Thus, in this case, it is not unreasonable to think that either the patient has a de novo variant or the variant comes from the deceased father, who passed away due to a brainstem stroke.

Regarding the biochemical profile, while in an international cohort of 990 patients with PCC, of whom $2 \%$ presented pathogenic TMEM127 germline variants, no preference of adrenaline or noradrenaline secretion was reported [8], three other studies have reported a predominant adrenaline secreting pattern $[7,13,14]$, which we have also described in this case, further raising the suspicion of an etiology distinct from $V H L$ or SDHB germline variants [15].

At present, the exact prevalence of TMEM127-related PCC is still unknown and will need to be defined by the TMEM127 genotyping of further large series. Moreover, due to the large number of genes responsible for the development of PCC, genetic testing remains a diagnostic challenge. In addition, it has been reported that $3 \%$ of apparently sporadic PCC were 

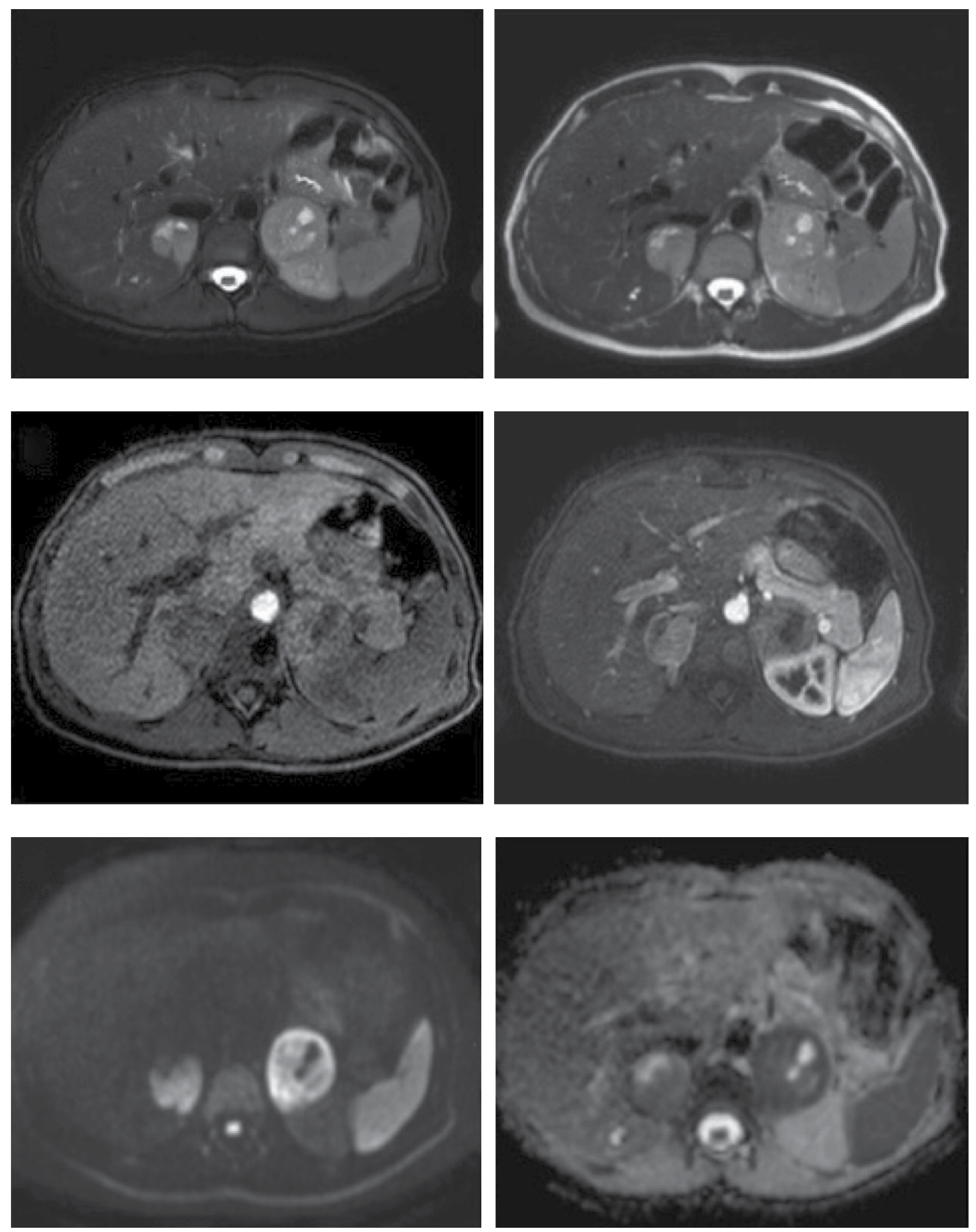

(c)

FIGURE 1: Abdominal MRI scan showing bilateral phaeochromocytoma. (a) axial TSE-T2W images with and without fat suppression; (b) axial GRE-T1W images prior to and $35 \mathrm{~s}$ after contrast injection; (c) DWI and ADC map showing restricted diffusion $\left(\mathrm{ADC}=0.6 \times 10^{-3} \mathrm{~mm} / \mathrm{s}\right)$.

caused by TMEM127 gene variants [4]. Taking into account the significant implications for patients and their family members, the identification of a possible underlying genetic variant is important. Thus, advances in DNA sequencing technologies that make possible simultaneous testing for multiple genes should be used. 


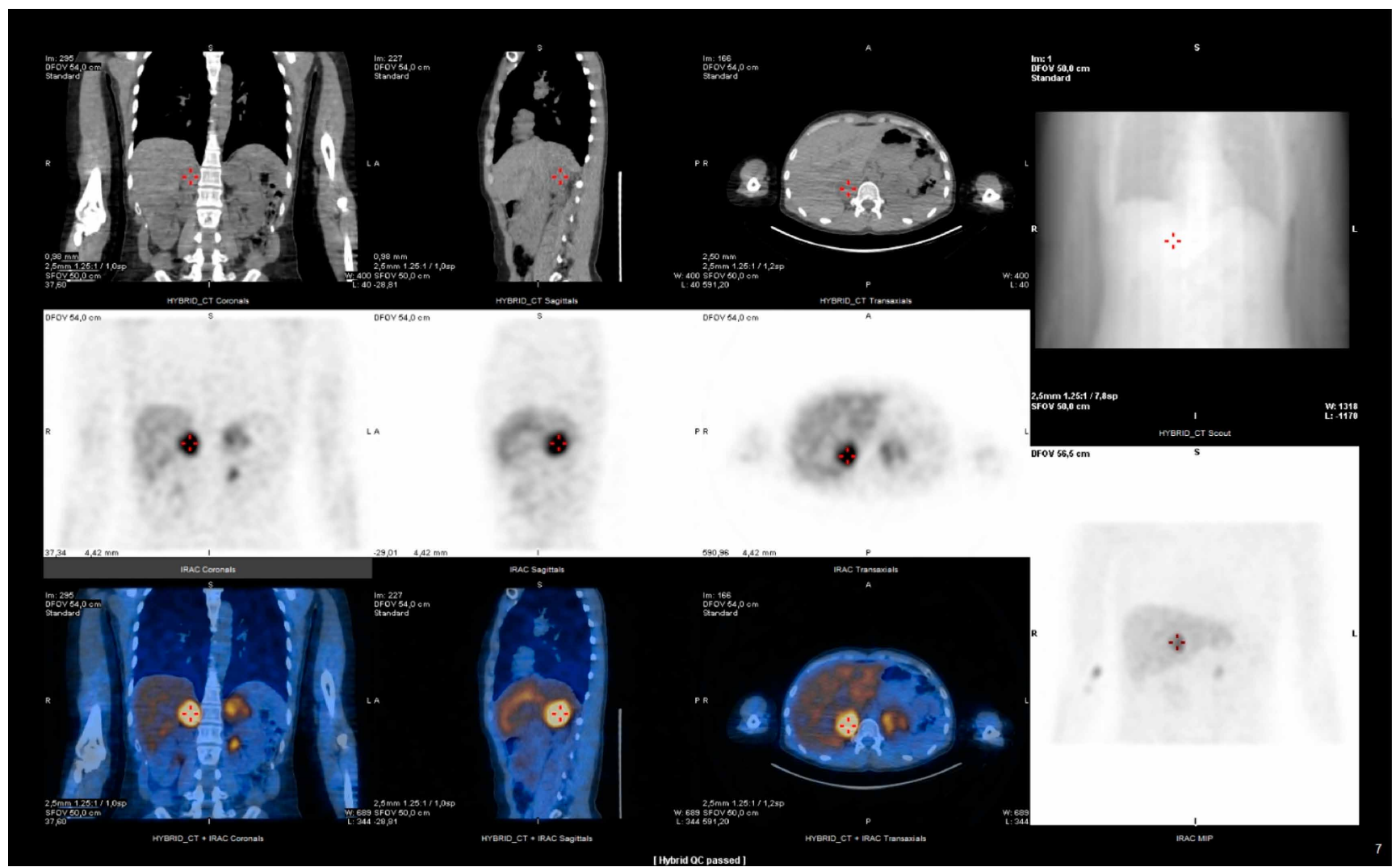

FIGURE 2: ${ }^{123}$ I-MIBG SPECT/CT showing increased uptake of the tracer in both adrenal glands, with right predominance.
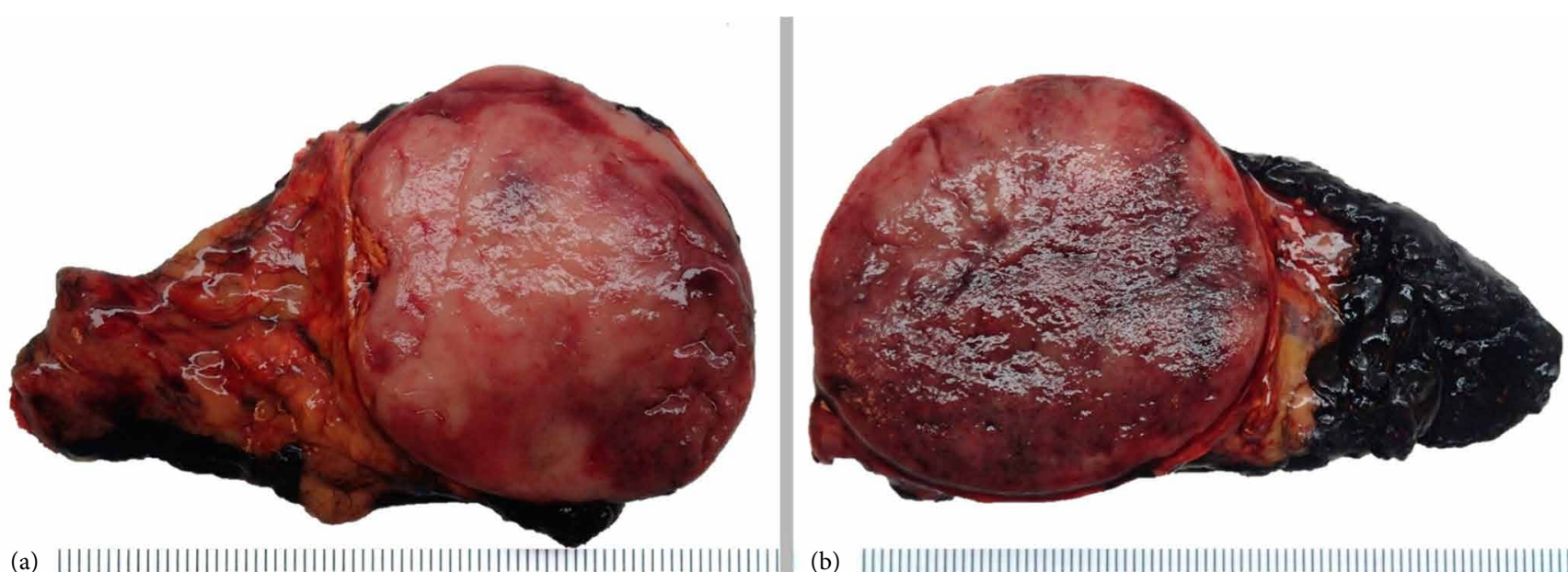

(a) ||||||||||||||||||||||||||||||||||||||||||||||||||||||||||||||||||||||||||||||

(b) ||||||||||||||||||||||||||||||||||||||||||||||||||||||||||||||||||||||||||||||||||||

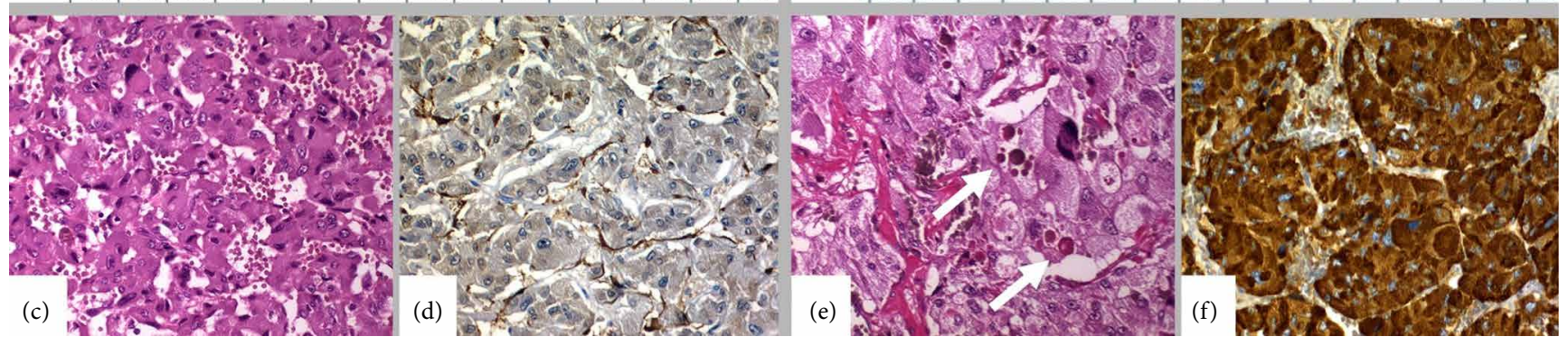

Figure 3: Bilateral phaeochromocytoma. Both right (a, c, d) and left (b, e, f) adrenal tumours were similar in the macroscopic and microscopic appearance. Microscopically they showed the typical nested pattern (c, e), moderate pleomorphism and occasional intracytoplasmic hyaline globules (arrows), but no mitotic activity. S100 sustentacular cells (d) and positivity for chromogranin A (f) were demonstrated. The Ki-67 was less than $2 \%$. 
This case supports the need to add TMEM127 to the multigene panel testing in patients with bilateral PCC with a predominant adrenaline secreting pattern, even if there is low suspicion of a hereditary condition.

\section{Data Availability}

The data that support the findings of this study are openly available in at https://databases.lovd.nl/shared/variants/ TMEM127/unique, reference number \#00234343.

\section{Consent}

Informed consent was obtained from the patient who participated in this study.

\section{Conflicts of Interest}

The authors declare that they have no conflicts of interest.

\section{Acknowledgments}

This work was funded by the Consellería de Industria, Xunta de Galicia (grant number ED341b 2017/19) (D.A-V), and by Grant PI15/0150-FEDER from the Instituto de Salud Carlos III, Ministry of Science, Innovation and Universities, Spain (JMC-T).

\section{References}

[1] A. S. Tischler, R. R. de Krijger, and A. Gill, "Phaeochromocytoma world health organization classification of tumours," in Pathology and Genetics of Tumours of Endocrine Organs, R. V. Lloyd, R. Y. Osamura, G. Klöppel, and J. Rosai, Eds., pp. 183-189, IARC Press, Lyon, 4th edition, 2017.

[2] H. P. Neumann, B. Bausch, S. R. McWhinney et al., "Germ-line mutations in nonsyndromic pheochromocytoma," New England Journal of Medicine, vol. 346, no. 19, pp. 1459-1466, 2002.

[3] D. Astuti, F. Latif, A. Dallol et al., "Gene mutations in the succinate dehydrogenase subunit SDHB cause susceptibility to familial pheochromocytoma and to familial paraganglioma," The American Journal of Human Genetics, vol. 69, no. 1, pp. 49-54, 2001.

[4] Y. Qin, L. Yao, E. E. King et al., "Germline mutations in TMEM127 confer susceptibility to pheochromocytoma," Nature Genetics, vol. 42, no. 3, pp. 229-233, 2010.

[5] I. Comino-Mendez, F. Gracia-Aznarez, J. Schiavi et al., "Exome sequencing identifies MAX mutations as a cause of hereditary pheochromocytoma," Nature Genetics, vol. 43, no. 7, pp. 663667, 2011.

[6] B. P. Lewis, R. E. Green, and S. E. Brenner, "Evidence for the widespread coupling of alternative splicing and nonsensemediated mRNA decay in humans," Proceedings of the National Academy of Sciences, vol. 100, no. 1, pp. 189-192, 2003.

[7] K. G. Hernandez, S. Ezzat, C. F. Morel et al., "Familial pheochromocytoma and renal cell carcinoma syndrome:
TMEM127 as a novel candidate gene for the association," Virchows Archiv, vol. 466, no. 6, pp. 727-732, 2015.

[8] L. Yao, F. Schiavi, A. Cascon et al., "Spectrum and prevalence of FP/TMEM127 gene mutations in pheochromocytomas and paragangliomas," JAMA, vol. 304, no. 23, p. 2611, 2010.

[9] S. P. Toledo, D. M. Lourenço, T. Sekiya et al., "Penetrance and clinical features of pheochromocytoma in a six-generation family carrying a germline TMEM127 mutation," The Journal of Clinical Endocrinology \& Metabolism, vol. 100, no. 2, pp. E308-E318, 2015.

[10] C. Bruch, N. Dagres, H. Wieneke, C. Herborn, K. Brandt-Mainz, and R. Erbel, "Sinus node dysfunction with intermittent sinus arrest and AV-nodal escape rhythm as the first manifestation of pheochromocytoma," Zeitschrift für Kardiologie, vol. 91, no. 1, pp. 81-87, 2002.

[11] D. H. Streeten and G. H. Anderson, "Mechanisms of orthostatic hypotension and tachycardia in patients with pheochromocytoma," American Journal of Hypertension, vol. 9, no. 8, pp. 760-769, 1996.

[12] T. Majic and V. Aiyagari, "Cerebrovascular manifestations of pheochromocytoma and the implications of a missed diagnosis," Neurocritical Care, vol. 9, no. 3, pp. 378-381, 2008.

[13] H. P. Neumann, M. Sullivan, A. Winter et al., "Germline mutations of the TMEM127 gene in patients with paraganglioma of head and neck and extraadrenal abdominal sites," The Journal of Clinical Endocrinology \& Metabolism, vol. 96, no. 8, pp. E1279-E1282, 2011.

[14] N. Abermil, M. Guillaud-Bataille, N. Burnichon et al., "TMEM127 screening in a large cohort of patients with pheochromocytoma and/or paraganglioma," The Journal of Clinical Endocrinology \& Metabolism, vol. 97, no. 5, pp. E805E809, 2012.

[15] G. Eisenhofer, J. Lenders, W. Timmers et al., "Measurements of plasma methoxytyramine, normetanephrine, and metanephrine as discriminators of different hereditary forms of pheochromocytoma," Clinical Chemistry, vol. 57, no. 3, pp. 411-420, 2011. 


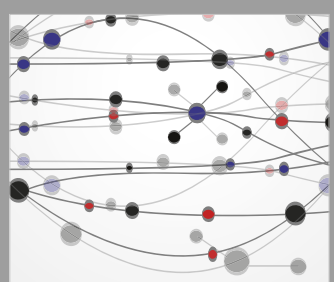

The Scientific World Journal
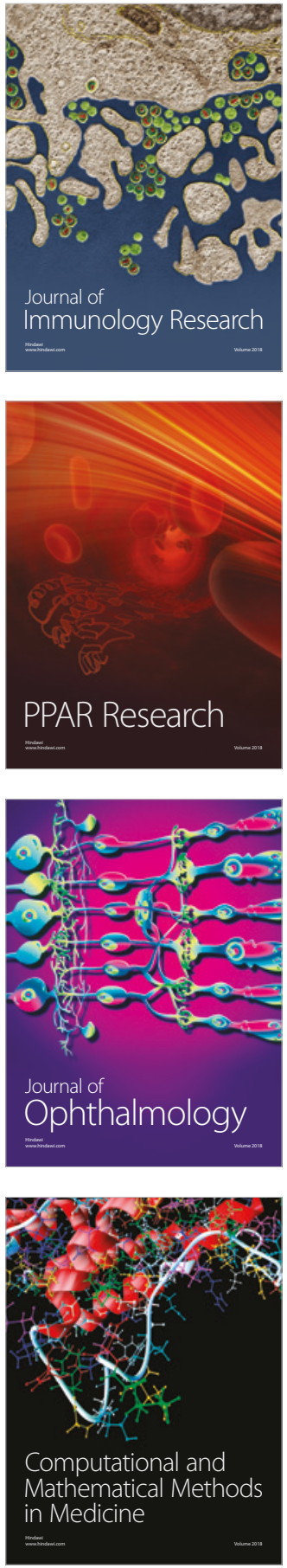

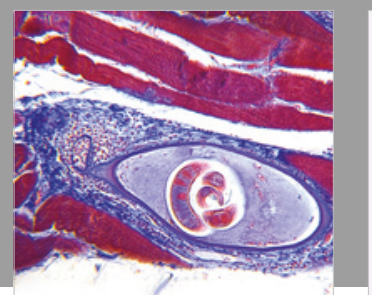

Gastroenterology Research and Practice

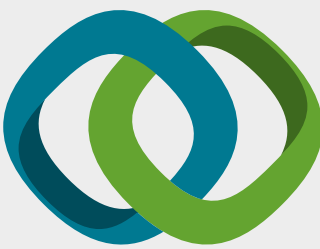

\section{Hindawi}

Submit your manuscripts at

www.hindawi.com
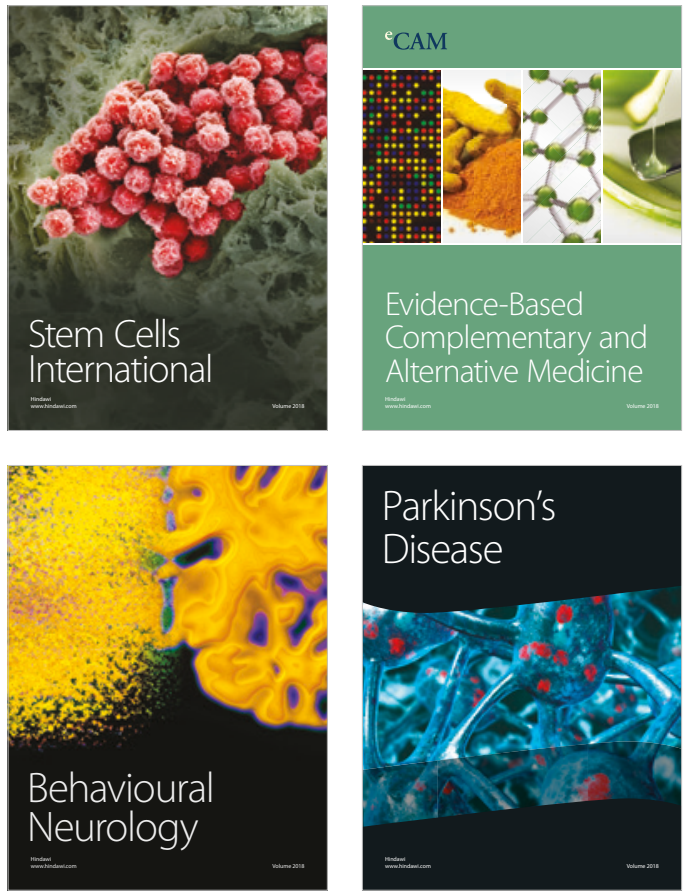

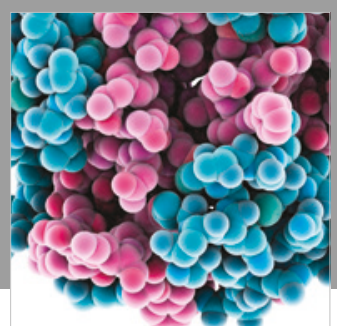

ournal of

Diabetes Research

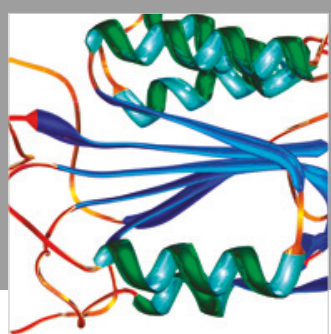

Disease Markers
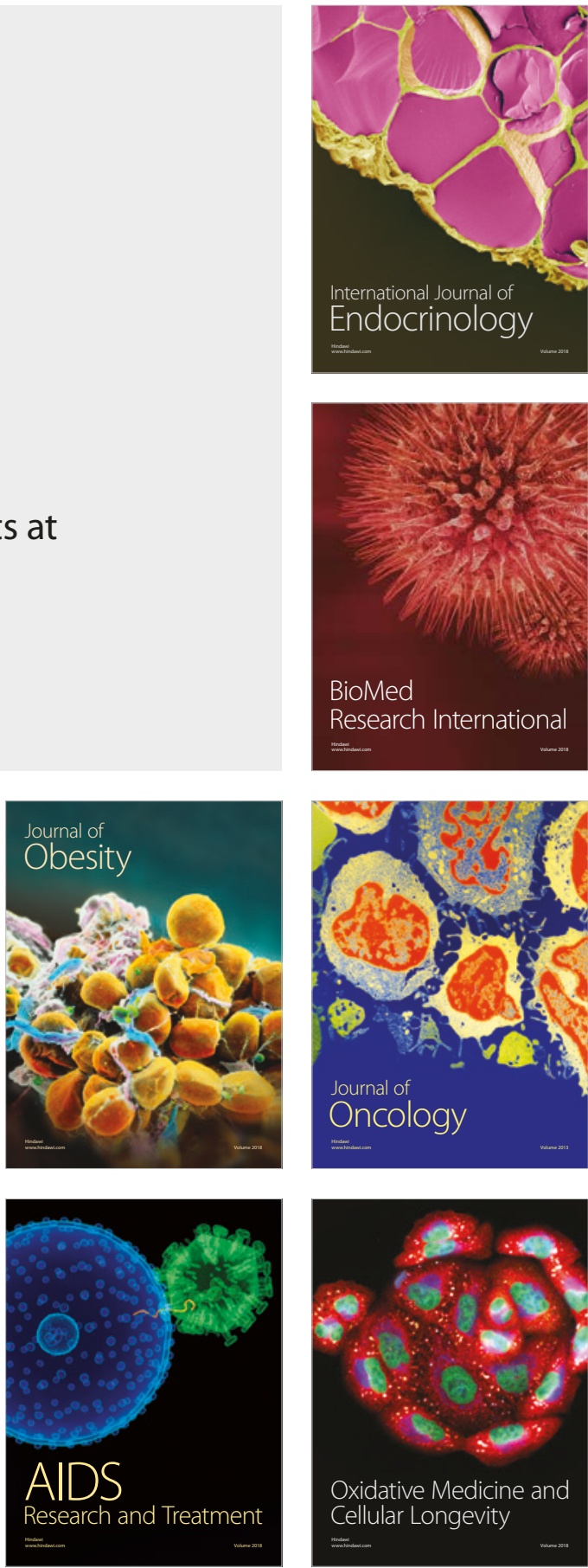